\title{
Estrategias de proximidad en las políticas asistenciales en Uruguay
}

\author{
Estratégias de proximidade políticas de assistência no Uruguai \\ Closeness strategies in social policies in Uruguay
}

\section{Carolina González Laurino* Sandra Leopold Costábile**}

\section{Resumen}

El acceso al bienestar implementado en Uruguay en los últimos 15 años prioriza líneas de universalización y focalización con pretensiones últimas de incorporación de la población vulnerable a los dispositivos universales y al trabajo formal. A partir de fuentes documentales y 60 entrevistas realizadas en 4 programas focalizados en la extrema pobreza, el artículo devela como estas "estrategias de proximidad" - con disponibilidad de escucha y acompañamiento, pero escasa materialidad disponible para responder a las necesidades de la población -, puede conducir a pequeñas transformaciones en la vida cotidiana de las personas, resultan insuficientes con relación a sus propósitos de inclusión social.

Palabras clave: Políticas asistenciales. Estrategia de proximidad. Integración social.

\section{Resumo}

O acesso ao bem-estar implementado no Uruguai nos últimos 15 anos prioriza linhas de universalização e focalização com o objetivo final de incorporar a população vulnerável em dispositivos universais e trabalho formal. A partir de fontes documentais e 60 entrevistas realizadas em 4 programas voltados para a pobreza extrema, o artigo revela como essas "estratégias de proximidade» -com disponibilidade de escuta e acompanhamento, mas pouca materialidade disponível para responder às necessidades da população-, pode levar a pequenas mudanças no dia a dia das pessoas, são insuficientes em relação aos seus propósitos de inclusão social.

Palavras-chave: Políticas de assistência. Estratégia de proximidade. Integração social.

\footnotetext{
* Doctora en Sociología y Ciencias Políticas, opción Sociología. Universidad de Deusto, España. Licenciada en Sociología. Universidad de la República. Licenciada en Trabajo Social. Universidad de la República. Docente e investigadora del Departamento de Trabajo Social de la Facultad de Ciencias Sociales de la Universidad de la República en Régimen de Dedicación Total. Coeditora de la revista Fronteras de la Facultad de Ciencias Sociales de la Universidad de la República. Co-coordinadora del Diploma en penalidad juvenil de la Facultad de Ciencias Sociales de la Universidad de la República. Investigadora activa del (SNI).

** Doctora en Ciencias Sociales con especialización en Trabajo Social. Universidad de la República. Magíster en Trabajo Social. Universidad Federal de Río de Janeiro y Universidad de la República. Especialista en Políticas Sociales. Facultad de Ciencias Sociales. Universidad de la República. Asistente Social. Universidad de la República. Docente e investigadora del Departamento de Trabajo Social de la Facultad de Ciencias Sociales de la Universidad de la República en Régimen de Dedicación Total. Co-coordinadora del Diploma en penalidad juvenil de la Facultad de Ciencias Sociales de la Universidad de la República. Coeditora de la revista Fronteras de la Facultad de Ciencias Sociales de la Universidad de la República. Investigadora activa del Sistema Nacional de Investigadores (SNI) de la Agencia Nacional de Investigación e Innovación (ANII).
} 


\begin{abstract}
Access to well-being implemented in Uruguay in the last 15 years prioritizes lines of universalization and targeting with the aim of incorporating the vulnerable population into universal devices and formal work. From documentary sources and 60 interviews conducted in 4 programs focused on extreme poverty, the article reveals how these "proximity strategies" - with availability of listening and accompaniment, but limited materiality available to respond to the needs of the population-, can lead to small changes in people's daily lives, are insufficient in relation to their purposes of social inclusion.
\end{abstract}

Keywords: Welfare policies. Proximity strategy. Social integration.

\title{
Introducción
}

El artículo surge de una investigación realizada durante el período comprendido entre diciembre de 2015 y diciembre de 2016 en el marco de un convenio entre el Departamento de Trabajo Social de la Facultad de Ciencias Sociales de la Universidad de la República y la Dirección Nacional de Evaluación y Monitoreo del Ministerio de Desarrollo Social uruguayo.

El estudio se centra en el análisis de las denominadas «estrategias de proximidad» desarrolladas en los programas interinstitucionales Cercanías, Jóvenes en Red, (URUGUAY, 2012) la Dirección Uruguay Crece Contigo y el Servicio de Abordaje Familiar del Ministerio de Desarrollo Social con el propósito de precisar sus definiciones, supuestos y modalidades de implementación.

La elección de estas propuestas se sustenta en su calificación como «programas prioritarios» por parte del Ministerio de Desarrollo Social en función de requerimientos de urgencia relacionados con los sectores de extrema pobreza y la especialización de la intervención.

El estudio pretendió obtener respuestas a las siguientes interrogantes: ¿Cuáles son las definiciones de la estrategia de proximidad en estas propuestas programáticas? ¿Qué supuestos dan fundamento a estas definiciones? ¿Cómo se expresan estas definiciones en los procesos de implementación de la estrategia de proximidad en los 
programas seleccionados? ¿Qué límites y posibilidades presenta la proximidad como estrategia de intervención?

Desde el punto de vista metodológico, el estudio se apoya en el análisis de fuentes documentales y del producto de 60 entrevistas realizadas en los departamentos de Montevideo y Salto a personas con distintos niveles de responsabilidad en cada propuesta (operadores y supervisores de todos los programas), así como a figuras específicas de cada estrategia: coordinadores (Servicio de Abordaje Familiar), referentes temáticos (Jóvenes en Red) y facilitadores (Uruguay Crece Contigo).

La muestra de entrevistados se seleccionó en base a criterios de representatividad y coexistencia institucional de los tres programas - Cercanías, Jóvenes en Red y la Dirección Uruguay Crece Contigo - en las ciudades de Montevideo y Salto. En el caso de Montevideo, el criterio tomó en cuenta la dimensión que alcanzan estas propuestas en la capital del país, además de considerar el Servicio de Abordaje Familiar que opera en Montevideo en la sede central del Ministerio de Desarrollo Social.

A los efectos de identificar el universo a entrevistar, en Montevideo se consideró la división geográfica de Este, Oeste y Centro del departamento, buscando el acceso, en cada segmento territorial, a dos equipos de cada programa territorial seleccionado, atendiendo, asimismo, a criterios de antigüedad en la gestión.

Tabla 1: Tabla de programas analizados

Estrategia Nacional de

Fortalecimiento de las

Capacidades Familiares. Cercanías.

\section{Propósitos}

Propiciar el acceso a prestaciones, derechos y servicios de familias en situación de extrema vulnerabilidad social.

Modalidad de intervención

El programa se organiza en torno a la conformación de dispositivos de cercanías - Equipos Territoriales de Abordaje Familiar (ETAF) - gestionados por organizaciones de la sociedad civil.

Cada equipo integrado por 2 duplas es responsable del acompañamiento de 15 familias durante 24 meses.

Organismos intervinientes MIDES, MSP, MTSS, MVOTMA, ANEP, ASSE, BPS, INAU.

\section{Entrevistados}

16 operadores: 6 psicólogos, 2 trabajadores sociales, 4 educadores sociales, 3 estudiantes de trabajo social y 1 de educación social.

4 supervisores: 3 psicólogos y 1 trabajador social. 
Jóvenes en Red

\section{Dirección Uruguay Crece Contigo}

Servicio de Abordaje Familiar

\section{Total: $\mathbf{2 0}$ entrevistas} Propósitos

Promover el ejercicio de derechos de los adolescentes y jóvenes de 14 a 24 años desvinculados del sistema educativo y el mercado formal de empleo.

\section{Modalidad de intervención}

El programa se organiza en torno a de equipos socioeducativos de 3 integrantes a los que se les adjudica la atención integral, territorial y en comunidad de 60 jóvenes durante 18 meses.

\section{Organismos intervinientes}

MIDES, MEC, MDN, MTYD, ANEP, UTU.

Entrevistados

12 operadores: 2 psicólogos, 4 maestros, 1 profesor de enseñanza media, 1 licenciado en educación física, 3 educadores sociales.

3 supervisores: 2 sociólogos y 1 psicólogo.

4 referentes temáticos: 2 psicólogos, 1 maestro y 1 trabajador social

\section{Total: 19 entrevistas}

\section{Propósitos}

Mejorar la salud, la nutrición, el desarrollo infantil y la inclusión social de mujeres embarazadas y niños menores de 4 años que viven en hogares de alta vulnerabilidad social, desde una perspectiva de derechos, género y generaciones.

Modalidad de intervención

El trabajo de acompañamiento socio- sanitario de cercanía es desarrollado de 6 a 9 meses,

por duplas integradas por un técnico del área de la salud y por otro del área social que realizan visitas en domicilio (entre 60 y 70 familias por dupla) con intensidad baja (visita mensual), media (quincenal) o alta (semanal).

\section{Organismos intervinientes MIDES.}

\section{Entrevistados}

11 operadores: 3 psicomotricistas, 2 psicólogos, 1 nutricionista, 1 enfermero, 1 trabajador social y 1 estudiante de trabajo social.

3 supervisores: 2 psicólogos y 1 nutricionista.

2 facilitadores: 1 nutricionista y 1 abogado.

\section{Total: 16 entrevistas}

\section{Propósitos}

Promover el acceso de las familias en situación de vulnerabilidad a las prestaciones sociales, fortaleciendo sus capacidades.

\section{Modalidad de intervención}

El Servicio se organiza entorno a duplas que acompañan a 40 familias en su domicilio, con una frecuencia quincenal y durante un tiempo no

preestablecido.

Organismos intervinientes MIDES.

\section{Entrevistados}

5 operadores: 3 psicólogos, 1 trabajador social y 1 estudiante de trabajo social. 
Fuente: Elaboración propia en función de los datos de la investigación. Ver siglas mencionadas en los documentos analizados listados al final del artículo.

\section{Contexto de diseño de la estrategia de proximidad en la política asistencial}

Entre las reformas impulsadas en los últimos 15 años en el país es posible enumerar la transformación de la matriz impositiva, la reforma del sistema de salud, la reinstalación de los Consejos de Salarios -que supone la negociación tripartita entre trabajadores, empresarios con mediación del Estado- y la creación del Ministerio de Desarrollo Social en 2005, como entidad responsable de la promoción y articulación de las políticas sociales a nivel nacional.

Las políticas sociales implementadas en el período 2005-2018 en Uruguay priorizan líneas de universalización en los sectores de salud y educación, así como de focalización mediante programas asistenciales dirigidos a la extrema pobreza.

El tipo de programas asistenciales objeto de estudio de la investigación constituye, en Uruguay, una estrategia que pretende intervenir sobre un sector de la población que no ha sido incorporado al mercado formal de empleo y permanece con serias dificultades para acceder al bienestar social, a pesar del crecimiento económico y el desarrollo social del país ${ }^{1}$ que supera la crisis del 2002, y continúa en ascenso con indicadores socioeconómicos favorables hasta $2014^{2}$.

Si bien en el año 2015 comienzan a percibirse socialmente los signos de un menor crecimiento, con aumento de la inflación, ${ }^{3}$ disminución de la tasa de empleo, ${ }^{4}$ indicadores negativos en la actividad industrial, y dificultades en los servicios universales como la

\footnotetext{
${ }^{1}$ Los datos del BANCO MUNDIAL (BM) presentan como principal logro del país la disminución de la pobreza que pasó de 32,5\% en el año 2006 a 9,7 en el año 2015. Los datos indicarían que la indigencia "prácticamente ha desaparecido reduciéndose del 2,5\% al 0,3\% para el mismo período". (BANCO INTERAMERICANO DE DESARROLLO, 2016).

2 De acuerdo al Ministerio de Economía y Finanzas (MEF), el Producto Bruto Interno durante el período 2005.2014 osciló entre 7,5\% en 2005 hasta llegar a un máximo de 7,8\% en 2010 y fue decreciendo en 2011 que marca un crecimiento del 5,2\% hasta el año 2014 que registra 3,2\%. El cambio que se produce en 2015 marca una tendencia a la baja que se inicia con un registro de 1\%. (URUGUAY, 2016b).

${ }^{3}$ De acuerdo a los datos del INSTITUTO NACIONAL DE ESTADÍSTICA, la evolución del Índice de Precios al Consumo en el total del país pasó de 68.64 \% en 2005 a 146.06 en 2015. (URUGUAY, 2016b).

${ }^{4}$ El buen desempeño macroeconómico también se reflejó en el mercado de trabajo que registró niveles de desempleo históricamente bajos en 2014 (6,6\%), aunque ante la marcada desaceleración del crecimiento, el mismo ha aumentado a $8.6 \%$ a julio de 2016. (BANCO INTERAMERICANO DE DESARROLLO, 2016).
} 
educación y la salud pública, los programas asistenciales analizados en este estudio persiguen el propósito del acceso a las prestaciones universales, algunos servicios específicos en temas de discapacidad o violencia doméstica, así como acceso a la educación y al mercado de trabajo formal en los sectores jóvenes y adultos que han desertado del sistema educativo o que nunca han accedido al mercado laboral, con el fin último de promover la integración social.

\section{Fundamentación de la modalidad de intervención en proximidad}

La estrategia de proximidad, enunciada como trabajo «cercano», "cara a cara», "cuerpo a cuerpo», que se desarrolla en el espacio donde transitan las familias y los jóvenes -con las particularidades que adquiere en cada uno de los programas analizados - parecería haberse instalado en las políticas asistenciales uruguayas sin mayores precisiones conceptuales, pero con el acuerdo implícito de que la población destinataria requiere de acciones de acompañamiento ${ }^{5}$ territorial que propicien el acceso al resto de la institucionalidad existente y al trabajo formal.

A pesar de las advertencias que las fuentes documentales del programa Cercanías exponen en relación a posibles efectos no deseados de la estrategia, como consecuencia de la sobre-representación de explicaciones [...] «psicologizantes» y/o «responsabilizantes» en detrimento de la valoración de las condiciones materiales de existencia de las familias (URUGUAY, 2015, p. 7), resulta posible pensar que los supuestos de partida de los programas estudiados, quedan atrapados en lógicas de individuación que participarán luego en los procesos de implementación y, por ende, en los resultados que se alcancen en relación a los objetivos enunciados.

Podría pensarse que este énfasis en la construcción del «puente» que la proximidad parecería tender a construir entre sujetos, protección social y mercado formal de trabajo, se posiciona desde una perspectiva que demanda intervenciones sociales

\footnotetext{
${ }^{5}$ Como analiza Bauman en su diagnóstico de la modernidad líquida, en la sociedad «pos-panóptica», que no depende de los enclaves de tiempo y espacio para la vigilancia, los pobres continúan siendo controlados mediante mecanismos que implican presencia y territorialidad (BAUMAN, 2006, p. 18).
} 
mediante un trabajo de subjetivación implicado en las políticas del individuo (MERKLEN, 2013, p. 77),

De un modo muy esquemático puede decirse que se pasó de un modelo «tutelar» [sintetizado en el modelo clásico de intervención del trabajo social] a un modelo que apunta a la «autonomía». Hay medidas concebidas ahora sobre la base del acompañamiento, la ayuda personalizada, la orientación, las formaciones, la proximidad, la participación... Se intenta movilizar a un individuo de quien se espera que establezca por sí mismo tanto los objetivos para salir de su dificultad como las estrategias más adecuadas para lograrlo (MERKLEN, 2013, p. 80).

Estas «políticas de individuación» que describe Merklen para el caso de las políticas asistenciales de «reparación» se centran en aquellos que se han caído y es necesario ayudar a levantarse. No, obstante, no cuestiona las reglas del juego ni se detiene a pensar por qué unos se caen y otros no, sino que "crean una nueva modalidad de políticas sociales tras haber completado una redefinición completa de las causas, efectos y posibles soluciones de los problemas sociales" (MERKLEN, 2013, p. 76).

Beneficiario y profesional están comprometidos en una relación de servicio que apunta a la definición conjunta (entre el beneficiario y el profesional de la ayuda) de la situación, de los objetivos a perseguir y de las estrategias de salida. La relación no solo se concentra en la situación singular del beneficiario, sino que lo busca activamente como sujeto de conocimiento y como principal recurso a movilizar. A esta movilización se refiere la categoría «activación» (MERKLEN, 2013, p. 82).

No obstante, no se trata de una tarea de restitución de derechos que parte de una deuda social, sino que las nuevas políticas de individuación piden al individuo que se «active» responsablemente y «devuelva» mediante una política de contraprestaciones lo que la sociedad parece haberle concedido como «apoyo, escucha y reparación» de una biografía que aparece como incompleta, donde el sujeto debe reconocer sus carencias y lo que ha hecho mal para encontrarse en la situación en la que ha caído. Detrás de [...] «la recitación perpetua» de su propia biografía, se sustenta la «sospecha» del «mal pobre», «que pesa sobre la cabeza del beneficiario, señalado como una persona vaga, perdedora, especuladora, perezosa. [...] (MERKLEN, 2013, p. 83). 
Como dice Castel en relación a la «individualización de las protecciones sociales» que se desarrollan en esta etapa de la modernidad, la interpretación que indica que el individuo gana libertad independizándose de las protecciones que lo sostienen, está basada en una «visión corta de la historia social», tanto como en «una visión ingenua del individuo» que se desdibujarían en la consideración de que las protecciones nacen con la modernidad en una relación «dialéctica» entre el individuo y el Estado. Por lo tanto, explica el autor,

Podría ser que la coyuntura más contemporánea exija no menores sino mayores protecciones. Tal vez sea paradójico, pero precisamente porque estamos cada vez más en una sociedad de individuos necesitamos cada vez más protecciones, porque para existir positivamente como individuo este necesita soportes protectores (CASTEL, 2010, p. 193-194).

Los discursos celebratorios del individuo liberado de los frenos burocráticos, jurídicos o estatales, advierte Castel, suponen un «no dicho». Se obvia mencionar que algunos individuos carecen de los soportes o recursos -que no son del orden de lo genético o de la voluntad-que les permitan responder favorablemente al anuncio de la libertad (CASTEL, 2010, p. 25).

Como indica Bauman, «el defender la autonomía humana y la libertad para elegir y autoafirmarse era el objetivo principal de la teoría crítica» (BAUMAN, 2001, p. 118). No obstante, se lamenta el autor, la época actual ha invertido sus objetivos (BAUMAN, 2001, p. 123) y actualmente nuestra crítica aparece, en sus palabras, "[...] «desdentada», imposibilitada de trascender el modelo autobiográfico, impelidos a elegir entre diversidad de posibles "proyectos de vida» (GIDDENS, 1994), "privatizada y desarmada» por la incapacidad de afectar «nuestras elecciones "político-vitales»" (BAUMAN, 2001, p. 115).

Es en este sentido que Fitoussi y Rosanvallón (2003, p. 36-37) hablan de la «ambivalencia del individualismo moderno» como "un vector de emancipación de los individuos, que incrementa su autonomía y hace de ellos sujetos portadores de derecho, y un factor de inseguridad que hace a cada uno más responsable de su porvenir y lo obliga a dar a su vida un sentido». Por eso, los procesos de individualización de la modernidad líquida, asegura Bauman implican «una autocrítica compulsiva» 
[...] ser individuo de iure significa no tener a nadie a quien echar la culpa por la propia desgracia, sino buscar las causas de sus propias derrotas en ninguna parte más que en la propia indolencia y pereza y no tratar de hallar otros remedios que esforzarse más y más (BAUMAN, 2001, p. 122).

Como indican Fitoussi y Rosanvallón (2003, p. 39) «Al mismo tiempo, la individualización-emancipación se acompaña con una individualización-fragilización», por lo que explica Bauman (2001, p. 122) "no es que no encuentren «soluciones biográficas» onerosas y engorrosas; es sencillamente que no hay "soluciones biográficas» a «contradicciones sistémicas»".

Por eso, de acuerdo con la interpretación de Bauman, la respuesta solo puede pasar por el vínculo del individuo con el Estado, a través del proceso ciudadano.

El «individuo de iure» no puede convertirse en «individuo de facto» sin convertirse primero en ciudadano. No hay individuos autónomos sin una sociedad autónoma, y la autonomía de la sociedad requiere una autoconstitución deliberada y reflexiva, que solo puede ser un logro conjunto de sus miembros (BAUMAN, 2001, p. 124).

He aquí la ambigüedad del proceso de individualización en la Modernidad que indicaban Fitoussi y Rosanvallón, "La reinvención positiva de un vínculo social más personal se acompaña insensiblemente con un mecanismo de desconexión, de «desafiliación», como diría Robert Castel” (FITOUSSI; ROSANVALLON, 2003, p. 37).

Reivindicando la potencia perdida de la política, Bauman lamenta que el «discurso ético-político de la sociedad justa» ha sido sustituido por el de los «derechos humanos», un paradigma que sostiene el derecho a la diferencia y a la búsqueda individual de la propia felicidad en la construcción de lo que Giddens (1994) Ilama un «estilo de vida» individualizado y privatizado (BAUMAN, 2006, p. 121).

\section{Implementación de la estrategia de proximidad}

La intervención de estos programas posibilita distinguir varios mojones en su implementación. Al inicio del trabajo se explica la propuesta (contenidos y duración), procurando el consentimiento de actuación de la población involucrada. Si esta fase 
prospera, se consensua un proyecto de trabajo y se establecen las metas a alcanzar, dando comienzo a la intervención. Esto derivaría en un «plan de seguimiento» en el que se enuncian los medios para alcanzar los fines con el apoyo para la progresiva autogestión por parte de la población. Este plan se implementaría mediante acciones de «acompañamiento social» centradas en la atención a las necesidades de los sujetos que participan de este tipo de programas. Sobre esta base se desarrollarían procesos particularizados con los individuos y/o familias, se fortalecerían los vínculos con la red primaria (familiares, allegados, recursos comunitarios), se facilitaría el acceso a los servicios sociales universales y se promovería el ingreso al mercado formal de empleo, en caso de corresponder.

Durante las primeras etapas del acercamiento y trabajo con la población objetivo de cada programa, los documentos y entrevistas enfatizan que la intervención requiere del acuerdo de los potenciales destinatarios y el establecimiento de un plan de trabajo que, en Jóvenes en Red, se realiza a través de un contrato escrito y firmado por ambas partes- para superar las dificultades visualizadas. En Cercanías, Servicio de Abordaje Familiar y Jóvenes en Red, este primer acuerdo de trabajo puede ser revisado y problematizado durante el proceso de intervención en caso de no cumplimiento o en situaciones donde los técnicos proponen otros aspectos a trabajar que no habían sido inicialmente incluidos. A diferencia de estos programas, en Uruguay Crece Contigo se enuncian con claridad los objetivos que se desarrollarán durante la intervención, ya que, en general, constituyen objetivos sanitarios fácilmente medibles, así como un trabajo sobre dificultades en el trato y la vinculación madre-hijo. Del material recogido en las entrevistas surge, generalmente, la aceptación de la propuesta de trabajo por parte de los destinatarios. Las excepciones que se mencionan en las entrevistas - sobre todo en Cercanías - aluden a familias multi-intervenidas o con situaciones complejas que han demandado la judicialización, en tanto en Uruguay Crece Contigo se hace referencia a familias que desarrollan actividades ilícitas. Sobre todo, los entrevistados afirman que las resistencias surgen en el desarrollo de los procesos de intervención y se manifiestan en términos de actuaciones o resistencias pasivas que no se verbalizan. Las negativas a la intervención propuestas son escasas en todos los programas. Tanto en Jóvenes en Red 
como en Uruguay Crece Contigo se asume que los jóvenes o las mujeres embarazadas y/o con hijos menores de 4 años a cargo continúan participando debido, tanto a las prestaciones materiales ofrecidas, como al valorado proceso de acompañamiento que despliegan los operadores desde estos dispositivos.

Una apuesta distinta es planteada por el programa Cercanías en el acceso a las prestaciones sociales que estarían, en principio, disponibles para el desarrollo del programa y a la que la inter-institucionalidad no parece responder. Los entrevistados refieren que, pese a los acuerdos institucionales firmados, las instituciones sectoriales participantes no comprometen recursos propios para la priorización de la población beneficiaria del programa. De esta forma, las coordinaciones interinstitucionales en territorio terminan dependiendo de la voluntad de técnicos y funcionarios, del conocimiento interpersonal y de las relaciones establecidas por el trabajo de los operadores en el territorio a lo largo del tiempo (BARÁIBAR RIBERO et al., 2015, 2016).

Estas observaciones colocan interrogantes acerca del alcance y la eficacia con la que operan los acuerdos intersectoriales en estos programas, máxime cuando el discurso de los actores entrevistados evidencia desconocimiento de los acuerdos existentes, así como insuficiencias en la calidad, cantidad y modalidad de gestión de las prestaciones y servicios que se disponen.

Parecería plantearse que los procesos significativos de carácter cualitativo solo parecerían posibles desde la excepcionalidad de los programas de proximidad, una excepcionalidad que resultaría difícil de encontrar en las prestaciones universales a la que la población sería derivada después de transitar por el acompañamiento cercano.

Sería conveniente problematizar en qué medida la existencia de estos programas, ha eliminado la proximidad que ya existía en algunas instituciones estatales. En este sentido, parecería oficiarse una cierta "especialización en proximidad», que puede terminar perjudicando el vínculo entre las instituciones y la población con vinculación previa. Salir de la institución a ver qué pasó con un joven o una familia que deja de concurrir a una propuesta de trabajo institucional, parecería que tiene que ser asumido por los programas de proximidad que son los que sabrían hacerlo, cuando, en general, las instituciones públicas tenían, en algún sentido, incorporadas esas funciones en su sistema 
tradicional de trabajo, sobre todo en relación con la educación y a la salud. Podría pensarse que el riesgo de construir un discurso sobre la excepcionalidad y originalidad de la proximidad supondría un mecanismo por el cual las instituciones sectoriales deleguen la responsabilidad del seguimiento de cierta población en los programas de proximidad. En tanto, así como desde los programas asistenciales se desarrolla un tipo caracterizado como «población MIDES», también parecería instalarse «un tipo de población que requeriría un acompañamiento cercano», que solamente sería posible de instrumentarse desde programas especializados en esta forma de relacionamiento (GONZÁLEZ LAURINO; LEOPOLD COSTÁBILE, 2018, p. 60-61).

A su vez, particularmente, la política asistencial expone severos inconvenientes para vehiculizar el tránsito de los sujetos participantes hacia el mercado formal de trabajo. Los operadores manifiestan problemas provenientes de la estigmatización de la que es objeto esta población por parte de los empleadores, así como insuficiencias en su nivel de formación e imposibilidad de sostener los requerimientos y las habilidades que el mercado formal de trabajo demanda. Con relación a las mujeres se agregan, además, dificultades para conciliar el trabajo con el cuidado de los hijos y las responsabilidades domésticas (BARÁIBAR RIBERO et al., 2015, 2016).

Finalmente, en el caso de los programas Cercanías y Jóvenes en Red, el tema del egreso genera una importante fuente de tensión entre los operadores, supervisores y la gestión del programa. Si bien se trata de programas acotados en el tiempo, cuya finalización se encuentra explícitamente prevista en la documentación, que sugiere plantearlo al inicio de la intervención, el egreso significa, tanto para las familias, para los jóvenes, como para los operadores una renuncia al logro de metas propuestas en el tiempo previsto. Sin embargo, aun suponiendo mayor frecuencia de contacto y un período de intervención más extendido en el tiempo, las situaciones problemáticas que estos programas buscan responder no encuentran espacio para su resolución mediante la focalización en las situaciones en las que la crudeza de la pobreza y la desigualdad social encuentra su expresión más dramática (GONZÁLEZ LAURINO; LEOPOLD COSTÁBILE, 2018, p. 58-59). 
No obstante, la proximidad no aparece cuestionada en las entrevistas de los técnicos, sino valorada como un trabajo necesario, cuyos objetivos serían imposibles de alcanzar de otro modo. Aunque se exponen algunas observaciones acerca de la intromisión en la vida cotidiana de la población objetivo o se cuestiona el acceso a la vivienda para conocer las características del relacionamiento familiar, la lógica del acuerdo de trabajo en la que se sustenta la intervención no registra cuestionamientos sustantivos en ninguno de los programas (GONZÁLEZ LAURINO; LEOPOLD COSTÁBILE, 2018, p. 61).

Las dificultades estarían colocadas en la implementación del trabajo en proximidad en función de tres conjuntos de problemas ya mencionados como: (i) la experiencia de sobrecarga en la tarea de los técnicos que visualizan como tensión entre la cantidad de población que se adjudica a cada dupla, el número de operadores a cargo de los supervisores, referentes temáticos y facilitadores y la calidad de la intervención en los distintos niveles de responsabilidad; (ii) la ausencia de medición y registro de los aspectos cualitativos de la intervención que se observaría en la tensión proceso-resultado, asociados al problema para el logro de objetivos programáticos a partir de las condiciones materiales y simbólicas de las familias y de las instituciones con las que se parte de un acuerdo político que no se concreta en la implementación, como fuera prometido; (iii) la ausencia de respuestas institucionales a las demandas activadas en la población a partir de la intervención en proximidad supone una exposición del operador que ofrece respuestas a las necesidades que no pueden satisfacerse mediante los recursos ofrecidos por la inter-institucionalidad. Esto genera una tensión entre las expectativas de la población y las posibilidades concretas de los operadores territoriales cuando tienen que dar cuenta de la carencia de recursos disponibles en términos de procesamiento en el tiempo, en cantidad y calidad ya referidos (GONZÁLEZ LAURINO; LEOPOLD COSTÁBILE, 2018, p. 61).

\section{Consideraciones Finales}

Si bien los programas asistenciales considerados, refieren, desde sus fuentes documentales y la palabra de sus actores, a la "proximidad» como herramienta específica 
de estos dispositivos, del análisis de estos discursos no surge una definición precisa y conceptualmente elaborada. La proximidad se propone como una estrategia que funcionaría como "puente» hacia las instituciones sectoriales que prestan servicios universales. En este sentido, la palabra de los operadores no se diferencia de la fundamentación programática.

Por otra parte, los discursos de los entrevistados, carentes de referencias precisas desde las fuentes documentales, mayoritariamente aluden a la proximidad a partir del relato de lo que efectivamente "se hace» en los procesos de intervención y la califican como un trabajo «mano a mano» y atención de "uno a uno». Por ello, resulta pertinente la observación de que la proximidad parecería «actuarse» más que conceptualizarse y, en este sentido, bien podría afirmarse que su esencia es «corporal». No obstante, y en concordancia con ciertos elementos mencionados en los documentos considerados, los enunciados exponen algunos componentes presentes en los procesos de intervención y que oficiarían como aspectos distintivos de la proximidad: la dirección que toma la intervención, el ámbito en el que se desarrolla, la duración y la frecuencia del contacto con el o los destinatarios, y la modalidad vincular que requiere.

Intervenir desde la proximidad en las propuestas programáticas consideradas, supone un accionar que, - parcialmente mencionado en las fuentes documentales, pero explícitamente enunciado en los discursos de los entrevistados - orienta la intervención desde la política a los destinatarios. En este sentido, la proximidad supone salir a la búsqueda y localización de uno o varios individuos, bajo el supuesto de que ellos no se movilizarán en dirección a los servicios sociales, educativos y/o sanitarios. Así, salir al encuentro de quién «no va a venir por sus propios medios», y hacia quién no se movilizará el resto de la institucionalidad pública, constituiría un sello distintivo de la proximidad en cuestión.

Esta dirección que adopta la intervención supone ir al ámbito socio-espacial donde los destinatarios se encuentran por lo que, el anclaje territorial y la acción en el hogar familiar asume relevancia primordial. La conceptualización del trabajo en proximidad supone cercanía «en el lugar donde transitan las personas», lo que genera un intercambio con otros vecinos del barrio e integrantes de la familia ampliada que ven en 
los programas una respuesta del Ministerio de Desarrollo Social a sus necesidades básicas. ${ }^{6}$ En este sentido, la estrategia de proximidad es concebida radicalmente opuesta al trabajo institucional tradicional o de «consultorio».

Algunos entrevistados relacionan la proximidad y el territorio de manera tal que solo sería factible concebir esta acción en tanto se desarrolle en el espacio que habita la población destinataria de cada propuesta programática. No obstante, puede afirmarse que varias de las instituciones mencionadas en forma reiterada por los entrevistados Centros de Atención a la Infancia y la Familia (CAIF), Escuelas, Servicios de Orientación, Consulta y Articulación Territorial (SOCAT), Policlínicas- hacia las cuáles se procura vincular a la población partícipe de estos programas, también ostentan cierto «anclaje territorial», en el sentido de operar con proximidad al lugar de residencia de los sujetos. Sin embargo, no parecerían ser percibidas por los entrevistados como dispositivos de tal naturaleza. Por ende, cabe formular la interrogante de si la territorialidad supone desde esta perspectiva, la acción de trasladarse - «de salir»- al ámbito donde están los destinatarios y no, forzosamente, la proximidad espacial en el territorio.

Tanto los documentos institucionales como el discurso de los operadores dan cuenta de que la modalidad de intervención en proximidad se desarrolla, fundamentalmente, dentro del hogar familiar en el caso de Cercanías y Uruguay Crece Contigo, y «en el lugar donde los jóvenes están» en el caso del programa Jóvenes en Red. El operar en el espacio físico dónde se desenvuelve la vida cotidiana, habilita la interrogante acerca de cuál es el conocimiento particular que se pretende alcanzar de los individuos y las familias involucradas que no se obtendría mediante otra modalidad de intervención y que parecería guardar exclusividad para esta política asistencial.

\footnotetext{
${ }^{6} \mathrm{Si}$ bien estos programas se conciben como intersectoriales, la proximidad parecería ser comprendida como acción exclusiva del Ministerio de Desarrollo Social y, en este sentido, podrían vivenciarse como intervenciones desligadas del resto de la matriz de protección social. Esto parecería ser resultado, entre otros factores, de una forma de presentación de los operadores que obviaría, por momentos, la mención al carácter intersectorial de cada programa como forma de facilitar los contactos iniciales y evitar la generación de expectativas, por ejemplo, en relación con la prestación social de la vivienda de difícil acceso, o deslindar la intervención de las representaciones sociales existentes en relación al Instituto del Niño el Adolescente en Uruguay.
} 
No obstante, es observable en el discurso de los entrevistados y en las fuentes documentales de Cercanías y Uruguay Crece Contigo un proceso de diversificación de la estrategia, que pasó progresivamente a considerar, más allá del hogar, otros espacios de intervención como ámbitos comunitarios o institucionales. La constatación empírica de que, en algunas situaciones, no estarían dadas las mejores circunstancias para intervenir en el hogar, ya sea por falta de condiciones adecuadas para el tratamiento de algunas temáticas específicas o porque se considera oportuno preservar la privacidad de las familias parecería haber propiciado esta modificación.

A su vez, la estrategia de proximidad supone tanto desde el diseño, como desde el discurso de los entrevistados, una modalidad de intervención que requiere de un tiempo de intervención y de una frecuencia en los contactos con la población de referencia. Más allá de las particularidades que asume esta dimensión en cada programa, puede observarse la noción de que la proximidad requiere de una asiduidad sostenida y una disponibilidad de atención que no se precisa en su alcance, aunque parecería concebirse sin interrupciones.

Este requerimiento, que parecería devenir en los procesos de intervención como resultado del tipo de relación que se establece entre quienes implementan cada programa y la población destinataria, traería consigo una sobrecarga en los operadores, obligados a estar atentos permanentemente a cada situación. ${ }^{7}$ A esta sobredemanda y disponibilidad permanente, además de las distancias a recorrer para acceder a determinados lugares de residencia, y la extensión de los territorios, se agrega la cantidad de individuos o familias asignados/as a cada equipo de trabajo territorial. Si bien esta variable experimenta diferencias en función de cada propuesta, e incluso a la interna de cada una de ellas, no deja de percibirse, en el discurso de los entrevistados, como un elemento que conspira

\footnotetext{
${ }^{7}$ En general, la estrategia de intervención en proximidad es positivamente valorada por los operadores que trabajan en territorio, tanto como por los supervisores que los acompañan. Resulta significativo, sin embargo, que, pese a la valoración positiva de la estrategia, los operadores coincidan en el nivel de desgaste humano que el trabajo en proximidad implica, refiriendo que «no es una actividad para toda la vida», que tiene un límite en las posibilidades de involucramiento, en el trabajo de "poner el cuerpo», la exposición del sí mismo como estrategia privilegiada de trabajo (GONZÁLEZ LAURINO; LEOPOLD COSTÁBILE, 2018, p. 61).
} 
contra la calidad de la intervención, dada la elevada magnitud que alcanza, con relación a los objetivos planteados y los recursos humanos disponibles.

En la mención explícita que fuentes documentales y entrevistados hacen acerca del «acompañamiento», este aparece, por un lado, asociado a aspectos muy concretos, como acompañar a las familias o a los individuos en la tramitación de documentación que abriría el acceso a servicios sociales disponibles para toda la población o un sector específico, coordinaciones, hospitalizaciones, consultas médicas, educativas y/o laborales. A su vez, refiere a la construcción de un vínculo interpersonal o «lazo de confianza» que difiere de la masividad y el trato impersonal de las instituciones clásicas. En este sentido, esta relación parecería concebirse desde los operadores consultados, en términos de horizontalidad.

Este tipo de vínculo interpersonal propiciaría una mayor apropiación del proceso de trabajo por parte de la población involucrada y parecería identificarse con un modo de atención que no se desarrollaría en otros ámbitos o políticas. A su vez, desde otra visión, se señala que también esa modalidad de relacionamiento propiciaría la construcción de subjetividades «agradecidas» (GRASSI, 2013, p. 281-282) en tanto cualquier tipo de modificación en las condiciones de vida es valorada como una «ayuda» que deviene del vínculo personal y exclusivo que se sostiene entre cada uno de los destinatarios y los operadores de cada propuesta en el marco de una institucionalidad programática que resulta difusa para sus destinatarios. Sin embargo, al mismo tiempo, esta forma de operar puede habilitar la «demanda» de vecinos y familiares que se interrogan acerca de por qué ellos no resultan seleccionados. En este sentido, las explicaciones del proceso de selección en la focalización resultarían tan opacas para los partícipes del programa, como para quienes no ingresan a las propuestas en consideración.

Las fuentes documentales consultadas, así como la palabra de los entrevistados de los programas, mencionan, fundamentalmente, tres aspectos que darían fundamento a esta modalidad de intervención: la existencia de un segmento poblacional «excluido», que conformaría un «núcleo duro de pobreza» en condiciones de "extrema vulnerabilidad», la imposibilidad o la dificultad que tendría esta misma población para acceder, por sus propios medios, a los servicios universales y al mercado formal de trabajo y la decisión 
pública de disponer acciones para salir a su encuentro, con propósitos de inclusión social, mediante la conformación de dispositivos exclusivamente generados para estos fines.

En este sentido, con ciertas variabilidades, sobre todo en lo que refiere a los objetivos a alcanzar, Jóvenes en Red, Cercanías, Uruguay Crece Contigo y el Servicio de Abordaje Familiar basan su accionar en torno al propósito de operar como nexo entre las familias y/o los individuos, el entramado de protección pública y el mercado de trabajo formal.

Los entrevistados reconocen la existencia de prácticas anteriores y actuales, ajenas a los programas en consideración, que podrían calificarse como estrategias de proximidad. Sin embargo, no se avanza en una explicación acerca de por qué, a pesar de contar con antecedentes institucionales, se han implementado nuevas propuestas programáticas, aun cuando se dirigen al mismo segmento poblacional. En este sentido, tampoco se visualiza una problematización acerca de la distinción entre estrategia y programa. Suponiendo que el trabajo de proximidad fuera necesario para responder a los objetivos planteados, no es claro por qué esto implica la necesidad de un nuevo dispositivo y no una acción que pueda desarrollarse desde la red pública existente.

Asimismo, la afirmación de que estos programas «llegan a lugares donde no llegaba nada» también constituye el discurso de los entrevistados quiénes, a su vez, señalan la preocupación porque este vínculo, en exclusividad, no devenga en dependencia. En este sentido, más que en la dificultad de relación entre la población, y la trama institucional y el mundo del trabajo formal, se observa un énfasis en la dimensión de lejanía de esta población, que no habría llegado a vincularse a los dispositivos universales y hacia la cual tampoco, ninguna propuesta programática se habría dirigido previamente. Esta valoración parecería desconocer el hecho de que, en más de un caso, la derivación a las propuestas asistenciales se realiza desde los servicios públicos existentes, a los que incluso el programa aspira a vincular o revincular, lo que cuestionaría la afirmación de que ninguna intervención se habría dispuesto previamente con relación a estas familias e individuos.

Paradójicamente, la trama institucional a la que se pretende relacionar a la población vinculada a estos programas es percibida por los entrevistados como 
«expulsora» de los sectores más vulnerables. Al mismo tiempo, los discursos de los operadores hablan de los escasos recursos disponibles en distintas áreas, entre las que se citan como ejemplo, el acceso a la vivienda para este sector de población, tratamiento para problemas de salud mental, discapacidad, adicciones, violencia doméstica, trata y tráfico de personas con fines de explotación sexual o situaciones de trabajo infantil que surgen como demandas para los operadores que actúan en territorio.

Si los «cupos» o «lugares prioritarios» no estarían dando preferencia a la población de estos programas, o los recursos son inexistentes, podría pensarse que, lo que no resiste al cuestionamiento, es la constitución de un «puente» hacia dichas prestaciones universales, específicas y al mercado de trabajo formal. En su defecto, funciona en mayor medida una especie de «movilidad lateral», como Merklen y Kessler (2013, p. 18) la denominan, ${ }^{8}$ que supone un tránsito de esta población de un programa asistencial a otro programa de similares características, con objetivos de educación o trabajo protegido, pero excepcionalmente, el acceso a un servicio de carácter compartido con el resto de la población.

Habría entonces que pensar cuál es la apuesta de la proximidad para trabajar con la población más pobre que transita por zonas social, económica, cultural y ambientalmente deterioradas si la inter-institucionalidad y la intersectorialidad que estas propuestas proponen acercar a estas personas no funciona como estaba inicialmente planteado. Estos cuestionamientos podrían verse acompañados, además, por los dilemas éticos que emergen en una intervención social que se ve expuesta sistemáticamente a la dificultad de concretar sus objetivos, y que coloca a los profesionales en testigos dolientes de las severas expresiones de la desigualdad social.

Dadas las dificultades señaladas, los entrevistados justifican la proximidad como resultado de la necesidad de concebir una intervención más específica, «individual» que, al tramitar las conexiones institucionales y laborales, desde un «vínculo» y un

\footnotetext{
${ }^{8}$ Los autores aluden a la conceptualización de Ruggiero y South (1997) y a Telles y Hirata (2010), aunque lo hacen para referirse al pasaje de los jóvenes de un empleo en situación de formalidad a una actividad informal o ilegal.
} 
«acompañamiento» con la población involucrada, posibilitaría la generación de propuestas de trabajo diseñadas de manera conjunta.

De esta manera, aunque la política asistencial tiene el propósito de contribuir a generar el nexo entre individuos y familias desafiliadas del resto de la institucionalidad existente y el trabajo formal, paradójicamente, la proximidad se focaliza en la población destinataria y parecería operar a distancia del entramado institucional y el mercado de trabajo. En este sentido, puede afirmarse que la estrategia de proximidad se dirige fundamentalmente a los individuos y familias, al tiempo que problematiza poco su accionar en relación con los funcionamientos institucionales y al mercado de trabajo. Los testimonios recabados exponen una conceptualización y una modalidad de intervención que hace foco en la construcción vincular con la población. Estos relatos propician la interrogante acerca de si esta priorización constituye una definición programática fundada o deviene como consecuencia de la escasa respuesta de las políticas públicas y el mercado de trabajo.

Este énfasis de la estrategia de proximidad que privilegia, en su accionar, a la población destinataria de la política asistencial en detrimento de los ámbitos institucionales y el mercado formal de empleo, contribuiría a reforzar la noción de que el desencuentro entre individuos, instituciones $y$ trabajo formal es responsabilidad fundamentalmente, sino en forma exclusiva, de los primeros. Sin constituirse en propósito expreso de estos programas, e incluso advirtiendo en algún caso de su riesgo, la intervención deviene en "política de individuación» (MERKLEN, 2013) y coloca bajo signos de interrogación el sentido de la proximidad y sus resultados (BARÁIBAR RIBERO et al., 2015, 2016).

\section{Referencias}

BANCO INTERAMERICANO DE DESARROLLO. Uruguay: panorama geral. 2016. Disponible en: http://www.bancomundial.org/es/country/uruguay/overview. Acceso em: 26 out. 2019.

BARÁIBAR RIBERO, X.; GONZÁLEZ LAURINO, C.; LEOPOLD COSTÁBILE, S.; PAULO BEVILACQUA, L. Las trayectorias de la inclusión como estrategias de intervención social. 
In: CUADERNOS de ciencias sociales y políticas sociales 2. Montevideo: Ministerio de Desarrollo Social, 2015. p. 7-52.

BARÁIBAR RIBERO, X.; GONZÁLEZ LAURINO, C.; LEOPOLD COSTÁBILE, S.; PAULO BEVILACQUA, L. El difícil tránsito hacia la inclusión social. De los dispositivos asistenciales hacia las políticas sectoriales universales y el mundo del trabajo. En: DERECHOS humanos en el Uruguay. Montevideo: Servicio de Paz y Justicia. 2016. p. 153-164.

BAUMAN, Z. La sociedad individualizada. Traducción: María Cóndor. Madrid: Cátedra, 2001.

BAUMAN, Z. Modernidad líquida. Traducción: Mirta Rosemberg en colaboración con Jaime Arrambide Squirru. Buenos Aires: Fondo de Cultura Universitaria, 2006.

CASTEL, R. El ascenso de las incertidumbres. Trabajo, protecciones, estatuto del individuo. Traducción: Víctor Goldstein. Buenos Aires: Fondo de Cultura Económica, 2010.

FITOUSSI, J. P.; ROSANVALLON, P. La nueva era de las desigualdades. Traducción: Horacio Pons. Buenos Aires: Manantial, 2003.

GIDDENS, A. Consecuencias de la modernidad. Traducción: Ana Lizón Ramón. Madrid: Alianza Editorial, 1994.

GONZÁLEZ LAURINO, C.; LEOPOLD COSTÁBILE, S. Transformaciones en la matriz de protección uruguaya a inicios del siglo XXI. Textos \& Contextos, Porto Alegre, v. 17, n. 2, p. 52-56, maio/ago. 2018.

GRASSI, E. El sujeto de la política social. Obstáculos persistentes y condiciones necesarias para el ejercicio de los Derechos, Revista SER Social, Brasília, v. 15, n. 33, p. 280-300, jul./dez. 2013.

KESSLER, G.; MERKLEN, D. Una introducción cruzando el Atlántico. In: CASTEL, R.; KESSLER, G.; MERKLEN, D.; MURAD, N. Individuación, precariedad, inseguridad. ¿Desinstitucionalización del presente? Buenos Aires: Paidós. 2013. p. 9-31.

MERKLEN, D. Las dinámicas contemporáneas de la individuación. In: CASTEL, R.; KESSLER, G.; MERKLEN, D.; MURAD, N. Individuación, precariedad, inseguridad. ¿Desinstitucionalización del presente? Buenos Aires: Paidós. 2013. p. 45-86.

RUGGIERO, V.; SOUTH, N. The late city as a bazaar. Drug, markets, illegal enterprise and barricade. British Journal of Sociology, London, v. 48, n. 1, p. 54-70, 1997.

TELLES, V. S.; HIRATA, D. V. Ilegalismos e jogos de poder em São Paulo. Revista Tempo Social, São Paulo, v. 22, n. 2, 2010. Disponible en: http://dx.doi.org/10.1590/S010320702010000200003. Acceso en: 16 abr. 2016. 
URUGUAY. Instituto Nacional de Estadística - INE. Índice de precios al consumo. 2016a. Disponible em: http://www.ine.gub.uy/ipc-indice-de-precios-al-consumo. Acceso en: 26 out. 2016.

URUGUAY. Ministerio de Economía y Finanzas. Producto bruto interno. 2016b. Disponible en: https://www.mef.gub.uy/10472/1/mef/producto-bruto-interno.html. Acceso en: 26 out. 2016.

URUGUAY. Modelo de atención cercanías. Montevideo: Ministerio de Desarrollo Social 2015.

URUGUAY. Propuesta jóvenes en red (diseño del programa): propuesta de elaboración interinstitucional. Montevidéo: Ministerio de Educación y Cultura, 2012.

Recebido em: 04/09/2018

Aceito em: 08/11/2019 